\title{
Review: incidence of dementia and Alzheimer's disease increases with increasing age
}

Jorm AF,Jolley D. The incidence of dementia: a meta-analysis. Neurology 1998 Sep;51:728-33.

\section{Question}

Are incidence rates of dementia related to age, sex, and national differences?

\section{Data sources}

A search was done in Medline (1966-97) for incidence studies and the bibliographies of relevant articles were reviewed.

\section{Study selection}

Studies were selected if the case finding was based on a field survey or study of medical and social agencies, age specific incidence rates of dementia were reported for age groups spanning $\leqslant 10$ years, the patient sample was population based, incidence rates were reported for mild or moderate dementia, and the SEs of the incidence rates were calculable.

\section{Data extraction}

Age specific incidence rates and SEs were extracted from each study. Other study features extracted were diagnosis (dementia, Alzheimer's disease, or vascular dementia), diagnostic criteria used (DSM-III-R, National Institute of Neurological and Cognitive Disorders and Stroke, or other), severity of dementia, study design, and region studied (Europe, United States, or East Asia). Age specific log incidence rates/1000 patient years were plotted and fitted with loess curves.

\section{Main results}

23 studies were included. For mild and moderate dementia, the age specific incidence rates increased exponentially with increasing age with no tendency to level off in old age ( $>85 \mathrm{y})$. The incidence rates were lower for East Asia than for Europe (age specific incidence rate for mild dementia at age 85 to $89 \mathrm{y}$, $72.1 v 104.1$ cases per 1000 person years, respectively, $\mathrm{p}<0.001$ ). For Alzheimer's disease the incidence rates also increased exponentially with age. Incidence rates varied across studies for vascular dementia. Region did not affect the incidence of Alzheimer's disease $(p=0.105)$ or vascular dementia $(p=0.85)$. No sex difference existed for the incidence of dementia $(p=0.21)$. A non-significant trend existed for older women to have a higher incidence of mild Alzheimer's disease and for younger men to have a higher incidence of vascular dementia.

\section{Conclusions}

The incidence of dementia and Alzheimer's disease increases exponentially with increasing age and shows no levelling off in old age. East Asia has a lower incidence of dementia than Europe. Older women tend to have a higher incidence of Alzheimer's disease and younger men tend to have a higher incidence of vascular dementia.

Source of funding: none.

For correspondence: Dr Anthomy Jorm, NHMRC Psychiatric Epidemiology Research Centre, Australian National Unizersity, Canberra, ACT 0200, Australia. Fax +61262490733.

\section{Commentary}

The measurement of the frequency of a disease is a basic prerequisite to understanding its cause. Until recently, most descriptions of the distribution of dementia in populations used prevalence as a measure of frequency. However, prevalence depends on the number of new cases of the disease and how long people survive. The incidence of a disease is far more useful for understanding aetiology. The review by Jorm and Jolley combines 23 studies to ask questions about the incidence of dementia in different age, sex, and regional populations-questions that at present cannot be answered by any single study, hence the need for a meta-analysis. The main finding is that the incidence of dementia increases with age with no decline in the rate of increase in older age groups.
Another meta-analysis addressing the same question by Gao et al was published in the same month. ${ }^{1}$ It found that, in contrast to Jorm and Jolley, the rate of increase in dementia slowed down with increasing age and that women were 1.5 times more likely to develop Alzheimer's disease than men. Why should 2 meta-analyses published at the same time come to different conclusions? Firstly, the 2 analyses used different criteria to select papers for inclusion. Jorm and Jolley used broader criteria for selecting studies. Gao et al restricted themselves to studies where only DSM-III or DSM-III-R diagnoses were made by personal interview and examination. This resulted in Jorm and Jolley having nearly twice as many studies in their analysis as Gao et al. Secondly, the statistical analyses used are different in the 2 meta-analyses and difficult for clinicians to interpret. At present, it seems that the jury is still out on the change of incidence with age and sex differences in Alzheimer's disease, although more weight should be given to the larger meta-analysis.

We can say that the incidence of the dementias increases with age, although it is not clear whether the rate of increase declines as people get older. Furthermore, if women have a higher incidence of the dementias than men then the difference is not great. Explanations of the causes of the dementias now need to incorporate both of these observations.

Simon Hatcher, MBBS, MMedSc University of Auckland Auckland, New Zealand

1 Gao S, Hendrie HC, Hall KS, et al. The relationships between age, sex, and the incidence of analysis. Arch Gen Psychiatry 1998;55:809-15. 